\title{
Not Obtained
}

National Cancer Institute

\section{Source}

National Cancer Institute. Not Obtained. NCI Thesaurus. Code C67325.

Not acquired, gained or come to the possession. 